\title{
Comparison of post operative outcomes in mandibular condylar fractures treated with single versus double non-compression mini plates.
}

\author{
Arjun Malhotra ${ }^{1}$, Rajeev Borle ${ }^{2}$, Nitin Bhola ${ }^{3}$, Bhushan Mundada ${ }^{4}$ \\ ${ }^{\text {I}}$ (Resident, Department of Oral and Maxillofacial Surgery,S.P.D.C,Sawangi(Meghe),Wardha,India) \\ 2 (Professor, Department of Oral and Maxillofacial Surgery,S.P.D.C,Sawangi(Meghe),Wardha,India) \\ ${ }^{3}$ (Associate Professor, Department of Oral and Maxillofacial Surgery,S.P.D.C,Sawangi(Meghe),Wardha,India) \\ ${ }^{4}$ (Assistant Professor, Department of Oral and Maxillofacial Surgery,S.P.D.C,Sawangi(Meghe),Wardha,India)
}

\begin{abstract}
The aim of this study was to compare the functions of the condyle and complications after fixation of a subcondylar fracture of the mandible with two noncompression miniplates and a single non-compression miniplate. A total of 24 patients who required open reduction of a subcondylar fracture of mandible were selected for the study. The patients were randomly divided into two groups of 12 each. Group I comprised of patients treated with a single miniplate and Group II were treated with two miniplates. The patients were assessed for occlusion, lateral deviation on opening, infection and plate removal.All the parameters were compared statistically using the chi square test. Out of 24 patients, inadequate reduction was noticed in two patients in Group I. Screw loosening occurred in two cases; both the cases were stabilized with a single miniplate. In these cases, hardware removal was performed. Malocclusion and lateral deviation occurred in three cases due to plate bending. When two miniplate were used, no screw loosening was observed. Malocclusion and inadequate reduction was observed in one of the patient in Group II due to plate bending. Two plates for subcondylar fractures represent the best solution to obtain stable osteosynthesis in comparison to a single miniplate.
\end{abstract}

Keywords: Miniplates, mandible fracture, malocclusion,noncompression, subcondylar fracture

\section{Introduction}

Although there is still controversy about therapy for condylar fractures in adult patients, many surgeons favor open treatment of displaced condylar fractures, because such reduction and rigid fixation allows for good anatomic repositioning and immediate function[1]. There are different treatment modalities mentioned in literature for the fixation of mandibular condylar fractures, in the form of wires, miniplates, lag screws,threedimensional plate, and the trapezoid plates and many more. Numerous methods of open reduction and osteosynthesis of condylar fractures have been described. However, very few studies have assessed plating techniques for such fractures. Hammer et al[2] reported on complications associated with different types of plates in fractures of the condyle. However, it was difficult to accurately assess treatment results of the different plating techniques because the number of cases in their study was very limited. Using an in vitro model, Choi et al[3] demonstrated that a 2-miniplate system applied to the anterior and posterior regions of the condylar neck was more stable than a single-plate system. Therefore, the goal of this study was to evaluate a sample of patients who had sub condylar fractures treated with 2 different plating techniques and to demonstrate which plating technique provides functionally stable fixation for fractures of mandibular sub condylar region.

\section{Materials And Methods}

This study was a randomized clinical trial. Approval for the present study was obtained from our institution's Experimental Medical Research and Practicing Center Ethical Committee. Informed consent was obtained from all patients who were enrolled in the study, after they received an explanation of the advantages and disadvantages of open and closed reduction in vernacular language. The study sample was derived from the population of patients who reported to the Department of Oral and Maxillofacial Surgery, Sharad Pawar Dental College (Wardha, India), between August 2011 and October 2013.

\subsection{Inclusion criteria :}

- Patients with a unilateral non-comminuted mandibular condyle fracture associated with symphysis and parasymphysis fractures of the mandible, and a midface fracture, reported within seven days for treatment.

- Patients had to be of age 18 years or older 


\subsection{Exclusion Criteria :}

- Patients unfit for surgery under general anesthesia

- Patients with history of occlusal disturbances or skeletal malocclusion

- Patients with insufficient dentition to reproduce occlusion.

All fractures were classified according to Spiessl and Schroll [4], into types I to VI [ Table 1]. Four fractures were classified as type II and twenty fractures as type III. The high condylar neck fracture was defined as a fracture with the fracture line extending over to the sigmoid notch. Low subcondylar fractures were defined as fractures of the condylar neck situated below a horizontal line drawn from the right to the left sigmoid notch on panoramic radiographs. Fourteen patients had an additional parasymphyseal fracture, six had symphysis fracture of the mandible, and four patients had associated midface fracture.

The patients were randomly divided into two groups of 15 each. Randomization was performed by lots in closed envelopes. Group I comprised of patients treated with a single miniplate and Group II were treated with two noncompression miniplates, in which one plate was fixed in a similar manner to that of Group I and the other plate was fixed medial to it, at a $5 \mathrm{~mm}$ distance [Figures 1 and 2].

A Risdon's incision was used to expose the fracture condyle and the intraoral vestibular incision, to expose the symphysis and parasymphysis fracture site. The fracture was then reduced and the jaws were placed into the intermaxillary fixation (IMF) with the help of arch bars / IMF screws. After placement of the plates, the IMF was released and the occlusion was checked. The intraoral incision was closed with resorbable sutures and the extraoral incision was closed in two layers (with 3-0 vicryl and 5-0 prolene), after securing the drain. Postoperatively, elastics were placed for one week, in every case. The patients were reviewed after surgery for six months. The elastics were removed after one week and the arch bars after the fourth week. A single surgeon performed all the surgeries. All patients, in both the groups, were given antibiotics (ampicillin $500 \mathrm{mg}$ intravenously four times a day, for five days postoperatively, and $1000 \mathrm{mg}$ intravenously, two hours before surgery.

The patients were assessed for malocclusion, lateral deviation on opening, infection, plate removal, facial nerve function as well as radiographic assessment of the fracture reduction was also done postoperatively [Figure 3 and 4].All the patients were assessed by a single assessor. Descriptive statistical analysis was performed with SPSS statistical software for windows, version 8.0 (SPSS, Inc, Chicago, IL).

\section{Results}

Out of 24 patients, 23 were male and 1 female. The cause of fracture in 17 patients was road traffic accident, 6 had a fall, and 1 patient was victim of assault. The mean age of the patients in both the groups was 29.6 (age range from 16 to 55 ).

Postoperatively, malocclusion was observed in one patient in Group II due to plate bending. Malocclusion and lateral deviation occurred in three patients in Group I, who had plate bending after fixation. These patient underwent functional treatment that consisted of tight mandibulomaxillary fixation (MMF) with elastic for 10 days, followed by active jaw exercises. Out of 24 patients, inadequate reduction was noticed in one patient in Group II and in three patients in Group I. Table 2 lists the complications encountered in both the groups.

Screw loosening occurred in two cases; both the cases were stabilized with a single miniplate. Screw loosening was always associated with chronic infection. In these cases, hardware removal was performed. Plate bending was observed in three case, which were stabilized with a single miniplate. When two miniplates were used, plate bending was observed in one case.Inadequate reduction was observed in two patients in Group I and in one patient in Group II.Malocclusion and lateral deviation was observed in three patients in Group I and in one patient in Group II.

\section{Discussion}

Problems with proper reduction and fixation of condylar fractures using a single miniplate have been discussed by various authors [5-7]. Hammer et al [2] reported that plate failure or screw loosening was observed in more than one third (35\%) of the group stabilized with a single adaptation miniplate. Others have also reported the fracture of miniplates used in condylar fracture fixation [8].

The goal of this study was to identify a better method of fixation after subcondylar fracture of the mandible. Specifically, the intent was to see the efficacy of two noncompression miniplates in comparison to a single noncompression miniplate in the fixation of a subcondylar fracture. The results of this study confirmed that two miniplates were better than a single miniplate for a fixation with less complication. Different approaches such as preauricular incision, endaural incision, a Risdon's incision, a submandibular incision, a retromandibular incision, the rhytidectomy approach, or an intraoral incision were mentioned in the literature, for exposing the condyle [5-7]. 
We used the Risdon's incision, without any complication, in all our cases, to expose the fractured condyle. The advantage of this approach was that we could pull the distal segment of the mandible downward by applying the bone holding forceps or a 24 gauge wire, which helped in reduction and adequate surgical access of the fractured condyle. Several complications are mentioned in the literature when a single miniplate is used for fixation of a condylar fracture. According to Hammer et al [2]. 35\% cases had plate failure or screw loosening when the fracture was stabilized, with a single miniplate. Sometimes the single plate also led to inadequate fixation.

We also observed plate bending (25\%) and screw loosening (16.66\%) in Group I and plate bending ( $8.33 \%$ ) in Group II of our study. Sargent and Green [8] also reported plate fracture in their study and they suggested that the functional forces exceeded the rigidity of one miniplate. To avoid plate fracture in cases of condylar fracture Ellis and Dean [5] used minidynamic compression plates, however they also reported bending of the plate and loosening of screws. Inadequate reduction occurred in two patients, lateral deviation on opening and malocclusion occurred in three patient, in whom the plate was bent, in Group I and no caseof inadequate reduction was observed in Group II whereas lateral deviation on opening and malocclusion was observerd in one patient, in whom the plate was bent. Infection occurred in two (13.33\%) of the cases, in whom screw loosening was present.

On the other hand, inadequate stability causing either plate fracture or screw loosening was not observed when two miniplates were used, which strongly suggested that two miniplates were better than a single miniplate for fixation. The second plate protected the first plate from the damaging mechanical strains that could cause its fracture, and prevented a secondary displacement of the mandibular condylar fragment [9] . According to Choi et al [10], the two-miniplate fixation technique provides functionally stable fixation for fractures of the condylar neck. They also suggest that application of a miniplate at the posterior and anterior borders of the condylar neck seem to have the beneficial effect of restoring tension and compression trajectories. Pilling et al [11], after comparative evaluation often different condylar base fracture osteosynthesis techniques, concluded that osteosynthesis with two miniplates would be the most stable way of treating a condylar fracture. Using an in vitro model, Choi et al [3], demonstrated that a two-miniplate system applied to the anterior and posterior regions of the condylar neck was more stable than a single-plate system.

\section{Conclusion}

The small sample size and limited follow-up could be considered the limitation of the study, but it is concluded from our pilot study results that the use of two plates for subcondylar fractures, represents the best solution to obtain stable osteosynthesis, in comparison to a single miniplate.

\section{References}

[1] Baker HW, Moos KF: Current consensus on the management of fractures of the mandibular condyle. Int J Oral Maxillofac Surg 27:258, 1998 .

[2] Hammer B, Schier P, Prein J: Osteosynthesis of condylar neck fractures: A review of 30 patients. Br J Oral Maxillofac Surg $35: 288$, 1997.

[3] Choi BH, Kim KN, Kim HJ, et al: Evaluation of condylar neck fracture plating techniques. J Craniomaxillofac Surg 27:109,1999.

[4] Spiessl B, Schroll K. Gelenkfortsatz- und Gelenkkoepfchenfrakturen. In: Nigst H, editor. Spezielle Frakturen- und Luxationslehre Bd. I/I. Stuttgart, Germany: Thieme; 1972.

[5] Ellis E, Dean J: Rigid fixation of mandibular condyle fractures. Oral Surg Oral Med Oral Pathol 76:6, 1993

[6] Klotch DW, Lundy LB: Condylar neck fracture of the mandible. Otolaryngol Clin North Am 24:181, 1991

[7] Undt G, Kermer C, Rasse M, et al: Transoral miniplate osteosynthesis of condylar neck fractures. Oral Surg Oral Med Oral Pathol Oral Radiol Endod 88:534, 1999

[8] Sargent LA, Green JFJ: Plate and screw fixation of selected condylar fractures of the mandible. Ann Plast Surg 28:235,1992.

[9] Parascandolo S, Spinzia A, Parascandolo S, Piombino P, Califano L. Two load sharing plates fixation in mandibular condylar fractures:Biomechanical basis. J Craniomaxillofac Surg 2010;38:385-90.

[10] Choi BH, Yi CK, Yoo JH. Clinical evaluation of 3 types of plate osteosynthesis for fixation of condylar neck fractures. J Oral Maxillofac Surg 2001;59:734-7.

[11] Pilling E, Eckelt U, Loukota R, Schneider K, Stadlinger B. Comparative evaluation of ten different condylar base fracture osteosynthesis techniques. Br J Oral Maxillofac Surg 2010;48(7):527-31. 


\section{FIGURES}

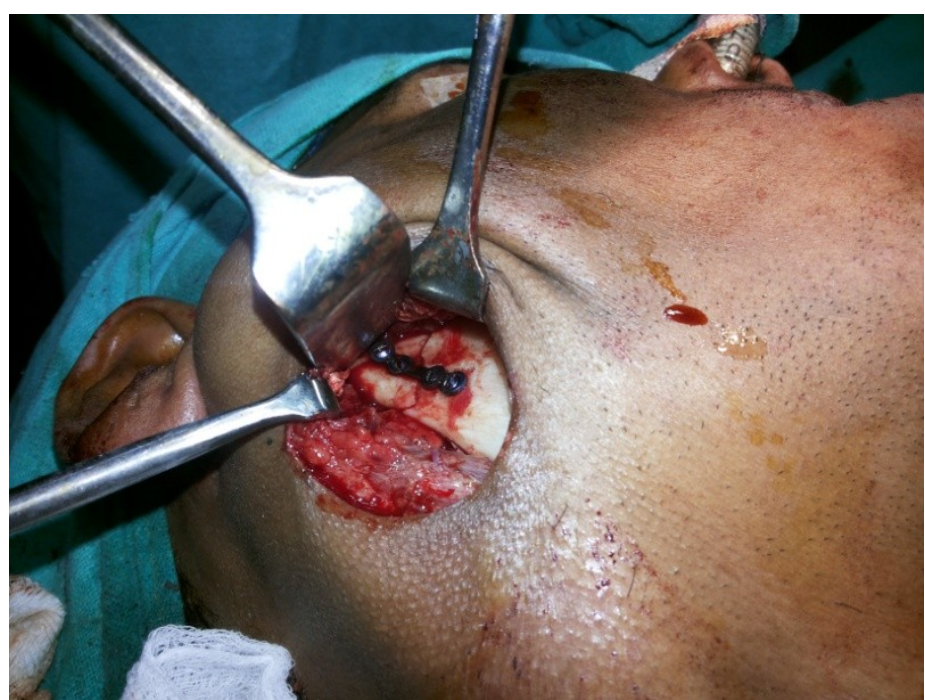

Fig 1 : Single miniplate fixation, Group I

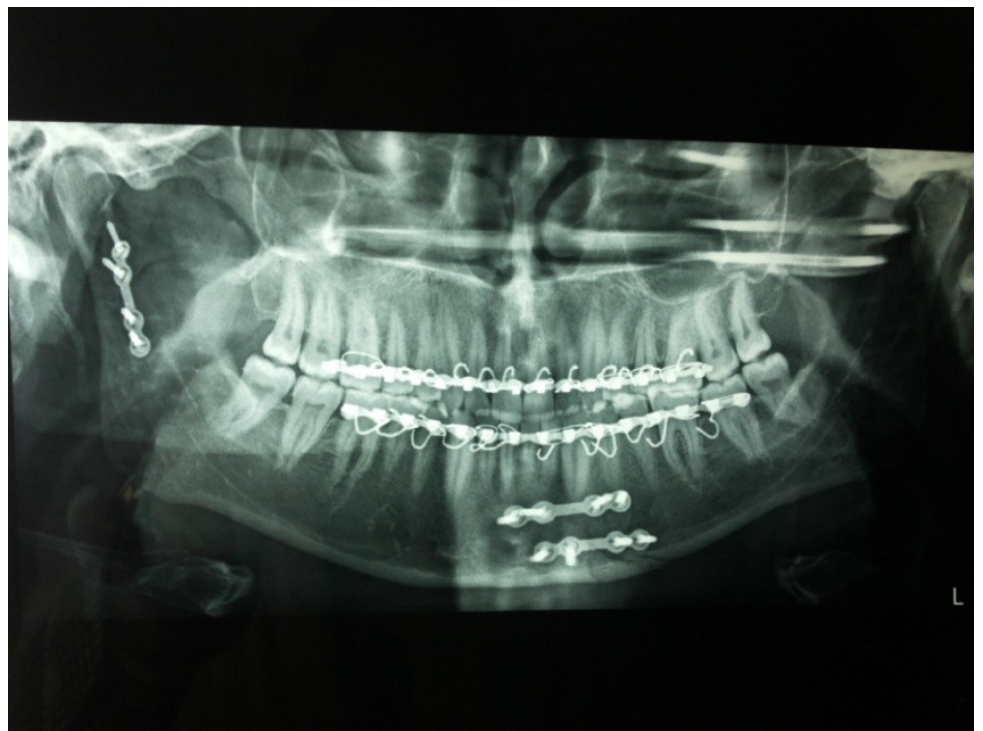

Fig 2 : Postoperative radiograph of patient in figure 1

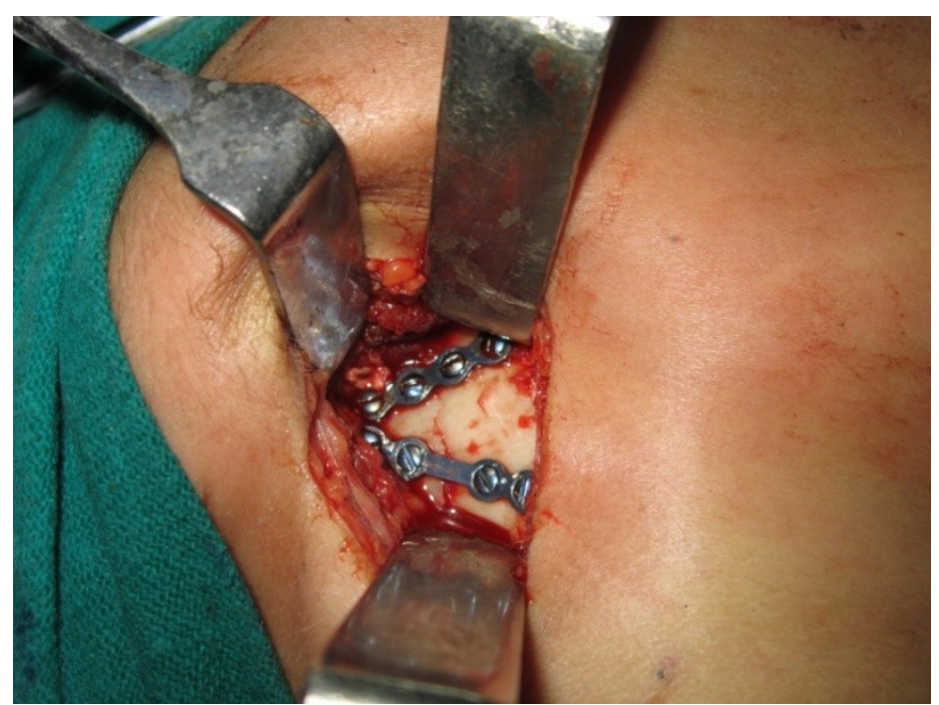

Fig 3 : Two miniplates fixation Group II 


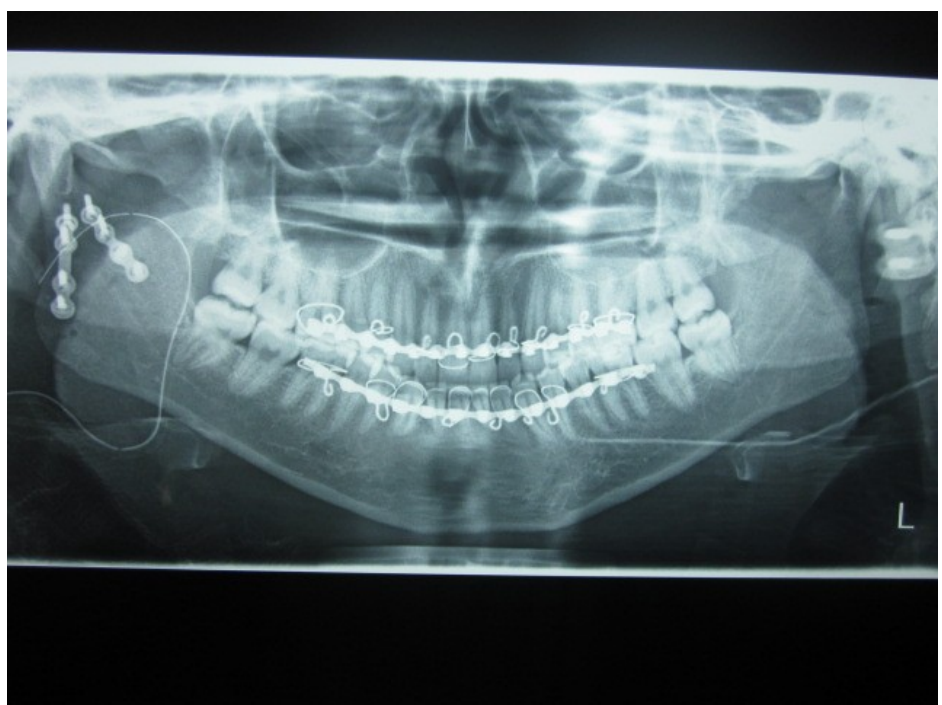

Fig 4 : Postoperative radiograph of patient in figure 3

\section{TABLES}

Table 1 : Spiessl and Schroll Classification of condylar fracture

\begin{tabular}{|ll|}
\hline Type I & Fractures without displacement \\
Type II & Low fractures with displacement \\
Type III & High fractures with displacement \\
Type IV & Low fractures with dislocation \\
Type V & High fractures with dislocation \\
Type VI & Intracapsular fractures \\
\hline
\end{tabular}

Table 2: Complications in both the groups

\begin{tabular}{lcc} 
Complication & Group I, n (\%) & Group II, n (\%) \\
\hline Inadequate reduction & $2(16.66)$ & 0 \\
Screw loosening & $2(16.66)$ & 0 \\
Plate bending & $3(25)$ & $1(8.33)$ \\
Malocclusion & $3(25)$ & $1(8.33)$ \\
Lateral deviation on opening & $3(25)$ & $1(8.33)$ \\
Infection & $2(13.33)$ & 0
\end{tabular}

